\title{
Preliminary Results from a Prospective Study Comparing White Blood Cell and Neutrophil Counts from a Laboratory to Those Measured with a New Device in Patients with Breast Cancer
}

\author{
ANNA C. LOHMAN ${ }^{1}$, INGRID VAN RIJN ${ }^{2}$, CHRISTINA L. LINDHARDT ${ }^{3}$, \\ REINHARD VONTHEIN $^{4,5}$, DIRK RADES ${ }^{6}$ and NIELS HENRIK HOLLÄNDER ${ }^{1}$ \\ ${ }^{1}$ Department of Oncology and Palliative Units, Zealand University Hospital, Naestved, Denmark; \\ ${ }^{2}$ Design School Kolding, Kolding, Denmark; \\ ${ }^{3}$ University College Absalon, Sor $\phi$, Denmark; \\ ${ }^{4}$ Institute of Medical Biometry and Statistics, ${ }^{5}$ Centre for Clinical Trials Lübeck, and \\ ${ }^{6}$ Department of Radiation Oncology, University of Lübeck, Lübeck, Germany
}

\begin{abstract}
Background/Aim: If blood tests were performed at home, unnecessary trips of patients for chemotherapy could be avoided. The HemoCue ${ }^{\circledR}$ WBC DIFF device was tested at home by 14 patients with breast cancer. Materials and Methods: A total of 42 measurements of white blood cell $(W B C)$ and neutrophil counts with the device at home were compared to laboratory measurements performed within 3 hours. Bland-Altman plots were created for limits of agreement that should be less than $1.0 \times 10^{9} / l$ for $W B C$ and $0.5 \times 10^{9} / \mathrm{l}$ for neutrophils to indicate a similar grade of intensity. Results: Limits of agreement were $-1.61 \times 10^{9} / l$ and $+2.34 \times 10^{9} / l$ for $W B C$ and $-1.15 \times 10^{9} / l$ and $+1.39 \times 10^{9} / l$ for neutrophils. All patients considered the device advantageous, particularly because they did not have to travel or wait for results. Most patients experienced problems with the lancet when taking blood samples. Conclusion: Disagreement of WBC and neutrophil counts between methods appeared clinically relevant. Findings need to be verified in a larger cohort, including the use of a different type of lancet.
\end{abstract}

In 2012, approximately 14.1 million people were diagnosed with cancer, and 8.2 million died from this disease (1). Many of these patients required systemic treatment, mainly

This article is freely accessible online.

Correspondence to: Niels Henrik Holländer, MD, Department of Oncology and Palliative Units, Zealand University Hospital, Rådmandsengen 5, 4700 Næstved, Denmark. Tel: +45 56513912, Fax: +45 56513269, e-mail: nhho@regionsjaelland.dk

Key Words: Breast cancer patients, white blood cell counts, neutrophil counts, chemotherapy, new device, preliminary results. chemotherapy. The treatment of patients with cancer is generally performed at specialized centers. This offers the patients the best available treatment. However, many patients live in smaller towns or the countryside and have to travel far to receive treatment at a specialized center.

One major side-effect of chemotherapy is bone marrow toxicity, resulting in leukopenia and neutropenia, that may not allow for administration of the planned treatment. In such a situation, the patients would have travelled a long distance, which may have taken up to several hours, in vain. For these patients, it would be of great value if they were able to measure their white blood cell (WBC) and neutrophil counts themselves at home before setting off for their scheduled chemotherapy session. A new device called HemoCue $^{\circledR} \quad$ WBC DIFF (Radiometer Medical ApS, Brønshøj, Denmark), which has been developed and CE-marked for the use by healthcare professionals, may be an option to help such patients. In a few studies, this pointof-care solution was found acceptably reliable and reproducible with a good correlation of up to $>0.99$ for the measurement of WBC and neutrophil counts when compared to standard methods (2-4). In another study comparing the HemoCue ${ }^{\circledR}$ WBC DIFF to venous samples analyzed at a laboratory, the correlation was less favorable for both WBC $(\mathrm{r}=0.77)$ and neutrophils $(\mathrm{r}=0.82)(5)$. Thus, more studies are required to evaluate further the potential value of this device during clinical routine. Currently, there is a lack of data regarding its usability for patients with cancer, particularly if the device is used by the patients themselves at home.

The present study compared the WBC and neutrophil counts measured at a university hospital laboratory to those measured with HemoCue WBC DIFF ${ }^{\circledR}$ at home by patients with breast cancer assigned to receive chemotherapy for nonmetastatic disease. 


\section{Materials and Methods}

Criteria for inclusion in this prospective study were non-metastatic breast cancer, assignment to chemotherapy, age of 18 years or older and written informed consent. Measurements were regarded evaluable for analyses when the time interval between the measurement of WBC and neutrophils counts at home and the measurement at the laboratory was less than 180 minutes. The study, which planned to perform 165 paired measurements in 33 patients, was approved by the local Ethics Committee (University Hospital Zealand, Naestved, Denmark). To date, 46 measurements for 14 patients have been analyzed. All patients received post-operative chemotherapy with three cycles of epirubicin $\left(90 \mathrm{mg} / \mathrm{m}^{2}\right)$ and cyclophosphamide $\left(600 \mathrm{mg} / \mathrm{m}^{2}\right)$ given every 3 weeks, followed by nine weekly cycles of paclitaxel $\left(80 \mathrm{mg} / \mathrm{m}^{2}\right)$.

The HemoCue ${ }^{\circledR}$ WBC DIFF represents a point-of-care solution based on cell counting using a micro-cuvette requiring a small volume of only $10 \mu \mathrm{l}$ of blood. The blood samples were taken by the patients from the fingertip using a sterile lancet (Vitrex Sterilance Press II; Vitrex Medical A/S, Herlev, Denmark). The first two drops of blood were not used for the measurements. The blood samples were drawn into the cavity of the micro-cuvette by capillary force. A cell-lysing agent hemolyzed the red blood cells and a staining agent stained the WBCs. The micro-cuvette was then placed in the analyzer and the number of WBCs was counted by image analysis. The HemoCue ${ }^{\circledR}$ WBC DIFF showed an error code if there was not enough blood in the cuvette. The measurements of WBC and neutrophil counts at the laboratory of the University Hospital in Naestved were performed with Sysmex XE-5000 and Sysmex DI-60 analyzers (Sysmex Corporation, Kobe, Japan). The repeatability coefficients of these analyzers were 0.06 for both WBC and neutrophil counts.

Initially, mean differences of the WBC and neutrophil counts between the measurements at the laboratory and with the HemoCue ${ }^{\circledR}$ WBC DIFF and the corresponding standard deviations were calculated. Based on these data, Bland-Altman plots were created for both WBC and neutrophil counts to calculate the limits of agreement and, additionally, identify a possible trend (increase or decrease of the difference between the methods with increasing mean difference) and inconsistency of the variability (increase in scattering with increasing mean difference) (6-8). For the BlandAltman plots, the mean value of both methods ([laboratory counts $+\mathrm{HemoCue}^{\circledR}$ counts] $/ 2$; x-axis) and the difference between both methods (laboratory counts - HemoCue ${ }^{\circledR}$ counts; y-axis) were calculated for each pair of measurements. In the plot, a point cloud of all 42 measurements was displayed. The limits of agreement are given by the average differences between the methods \pm 1.96 times the standard deviation of the differences. In addition, the coefficient of correlation was determined to compare the findings of the present study to the data from literature.

The latest version of the Common Terminology Criteria for Adverse Events (CTCAE), versions 5.0, was published in 2017 (9). The grading of leukopenia and neutropenia according to CTCAE is important when making the decision whether chemotherapy can be safely administered or would be too dangerous for the patient. According to CTCAE, WBC of $<3.0 \times 10^{9} / 1,<2.0 \times 10^{9} / 1$ and $<1.0 \times 109 / 1$ are defined as grade 2 (moderate), grade 3 (severe) and grade 4 (life-threatening) toxicity, respectively. Neutrophil counts of $<1.5 \times 10^{9} / 1,<1.0 \times 10^{9} / 1$ and $<0.5 \times 10^{9} / 1$ are defined as grade 2 , grade 3 and grade 4 toxicity, respectively. Steps between the different toxicity grades are $1.0 \times 10^{9} / 1$ for leukopenia and $0.5 \times 10^{9} / 1$ for neutropenia. Therefore,

HemoCue ${ }^{\circledR}$ WBC DIFF and laboratory were considered appropriately comparable for clinical purposes when the limits of agreement were within $\pm 1.0 \times 10^{9} / 1$ and for leukopenia and $\pm 0.5 \times 10^{9} / 1$ for neutropenia.

In addition, interviews were performed with all 14 patients. Before using the HemoCue ${ }^{\circledR}$ WBC DIFF, the patients and some of their closest relatives received an introduction and intensive training regarding the operation of this device. Furthermore, the patients had the option of contacting a specific telephone hotline if they had questions regarding the device and its operation. During the interviews, patients were asked about their experience with the HemoCue ${ }^{\circledR}$ WBC DIFF, including advantages/disadvantages, the role of their relatives, the value of introduction and training, and the use of the telephone hotline (see Table I).

\section{Results}

A total of 46 measurements of WBC and neutrophil counts were performed with the HemoCue ${ }^{\circledR}$ WBC DIFF by 14 patients (median age $=57$ years, range $=32-72$ years) at their homes and compared to 46 measurements for the same patients at the laboratory of the University Hospital Zealand in Naestved resulting in 46 measurement-pairs. The interval between paired measurements was less than 180 minutes for 42 measurements (median $=69$ minutes, range $=7-158$ minutes), which therefore qualified for the analyses. For these 42 paired measurements, the median number of paired measurements per patient was 3 (range=1-6). In the 11 patients with more than one paired measurement, the median time between two paired measurements per patient was 3 weeks (range=0.5-6.5 weeks). Per patient, the median maximum difference in WBC counts was $2.1 \times 10^{9} / 1$ (range $\left.=0.1-6.4 \times 10^{9} / 1\right)$ for the counts from the laboratory and $1.6 \times 10^{9} / 1$ (range $\left.=0.9-3.7 \times 10^{9} / 1\right)$ for the counts from the HemoCue ${ }^{\circledR}$ WBC DIFF at the patients' homes. The median maximum difference in the neutrophil counts was $1.4 \times 10^{9} / 1$ (range $0.1-4.6 \times 10^{9} / 1$ ) for those obtained at the laboratory and $1.3 \times 10^{9} / 1$ (range $0.5-3.4 \times 10^{9} / 1$ ) for those obtained with the HemoCue ${ }^{\circledR}$ WBC DIFF. Considering the relatively long median interval between two paired measurements per patient and the median maximum difference between the cell counts per patient, which was greater than $1.0 \times 10^{9} / 1$ for WBC and greater than $0.5 \times 10^{9} / 1$ for neutrophils, the different paired measurements of each patient were considered independent.

The mean WBC count for the 42 measurements performed at the laboratory was $4.65 \times 10^{9} / 1$ (Table II). Regarding the WBC counts, the mean difference between the two methods was $0.36 \times 10^{9} / 1$, and the standard deviation of differences was $1.01 \times 10^{9} / 1$. These data resulted in limits of agreement were $-1.61 \times 10^{9} / 1$ and $+2.34 \times 10^{9} / 1$ (Figure 1), while the correlation was 0.86 . Three of the 42 measurement-pairs $(7.1 \%)$ were outside the limits of agreement. In the plot, a 
Table I. Questions of the interviews performed with the patients after using the HemoCue ${ }^{\circledR}$ WBC DIFF device.

1. How well did you manage using the HemoCue ${ }^{\circledR}$ WBC DIFF at home?

2. What was the role of your relatives?

3. How was your experience regarding the introduction to the HemoCue ${ }^{\circledR}$ WBC DIFF and the training about using the device?

4. Have you used or needed the hotline?

5. What was your experience using the HemoCue ${ }^{\circledR}$ WBC DIFF at your own home?

6. Which advantages and disadvantages did you experience with taking a blood test yourself at your own home?

significant trend and inconsistency of the variability across the plot were not observed.

The mean neutrophil count for the 42 measurements performed at the laboratory was $2.32 \times 10^{9} / 1$ (Table II). Regarding the neutrophil counts, the mean difference between the two methods was $0.12 \times 10^{9} / 1$, and the standard deviation of differences was $0.65 \times 10^{9} / 1$, resulting in limits of agreement of $-1.15 \times 10^{9} / 1$ and $+1.39 \times 10^{9} / 1$ (Figure 2), while the correlation was 0.87 . Two measurement pairs $(4.8 \%)$ were outside the limits of agreement. A significant trend and inconsistency of the variability were not observed.

All 14 patients agreed to participate in the interviews, which were performed by professional staff members from the Design School Kolding and the Zealand University Hospital in Naestved. Twelve patients did the tests with the HemoCue ${ }^{\circledR}$ WBC DIFF themselves, and two patients were supported by their husbands. All patients were very positive about the instruction sheet provided with the device, which they found easy to understand. A few patients would have liked more thorough instruction and training regarding the use of the lancet, and one patient a better explanation of numbers and meaning of the normal values for WBC and neutrophils. Several patients called the telephone hotline once. When using the HemoCue ${ }^{\circledR}$ WBC DIFF, almost all patients experienced some problems with the lancet, which did not function properly when taking the blood samples. An error code occurred during the measurements for four patients. One patient felt stressed when the device showed an error code, and one patient did not feel completely safe because of using a new device. When asked about advantages and disadvantages of the HemoCue ${ }^{\circledR}$ WBC DIFF, all patients considered its use at home a great advantage when compared to the laboratory tests. Performing the measurements at home saved a lot of time, as the patients did not have to travel to the laboratory or to chemotherapy appointments if their counts were insufficient. Furthermore, some patients felt very positive about the fact that when using the HemoCue ${ }^{\circledR}$ WBC DIFF they received their results immediately. None of the patients saw any relevant disadvantages of the device. One patient mentioned that it required a lot of space, and some patients were a bit worried
Table II. Mean values and ranges of white blood cell and neutrophil counts obtained from the HemoCue ${ }^{\circledR}$ WBC DIFF and the laboratory.

\begin{tabular}{lcl}
\hline Cell type & $\begin{array}{c}\text { HemoCue }{ }^{\circledR} \text { WBC DIFF } \\
\text { Mean (range) }\end{array}$ & $\begin{array}{c}\text { Laboratory } \\
\text { Mean (range) }\end{array}$ \\
\hline White blood cells, $\times 10^{9} / 1$ & $4.28(1.7-12.7)$ & $4.65(1.9-11.0)$ \\
Neutrophils, $\times 10^{9} / 1$ & $2.19(0.7-8.1)$ & $2.31(0.6-6.2)$ \\
\hline
\end{tabular}

because the counts of both methods did not match as they had expected.

\section{Discussion}

A considerable number of patients with non-metastatic breast cancer require several cycles of chemotherapy that often causes bone marrow toxicity, including leukopenia and neutropenia (10-13). When leukopenia and neutropenia become more severe, continuation of the chemotherapy protocol may not be possible; chemotherapy may have to be postponed or even discontinued. Occasionally, patients have to travel a long distance to reach the center where chemotherapy is administered. Often WBC and neutrophil counts are measured only after the patient arrives at the center. In the case of significant leukopenia and neutropenia, the patient is sent home without chemotherapy after prolonged travel (10-13). In order to avoid this dissatisfactory situation, it would be helpful to be able to measure WBC and neutrophil counts at the patients' homes. This may be possible with a comparably new device, a pointof-care solution named HemoCue ${ }^{\circledR}$ WBC DIFF, which can measure both WBCs and neutrophils (2-5). Since it was developed to be used by healthcare professionals, there is a lack of data regarding the usability of this device at the homes of patients with cancer.

In the present study, we compared the leukocyte and neutrophil counts measured at the patients' homes with the HemoCue ${ }^{\circledR}$ WBC DIFF to the counts obtained from the 


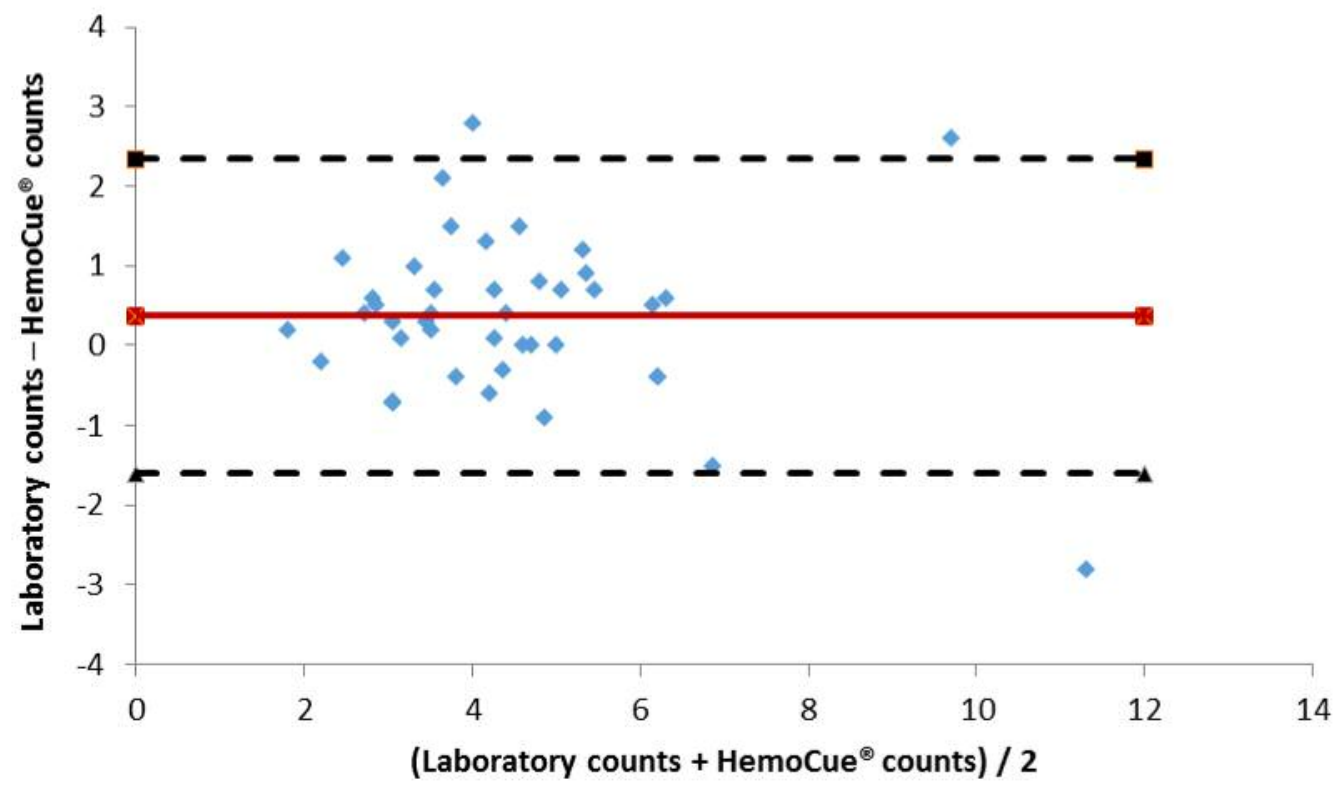

Figure 1. Bland-Altman plot of agreement of white blood cell (WBC) counts between HemoCue WBC DIFF $F^{\circledR}$ and the University Hospital laboratory. Dashed lines represent the limits of agreement (average difference \pm 1.96-times the standard deviation of the difference), the red line indicates the mean difference.

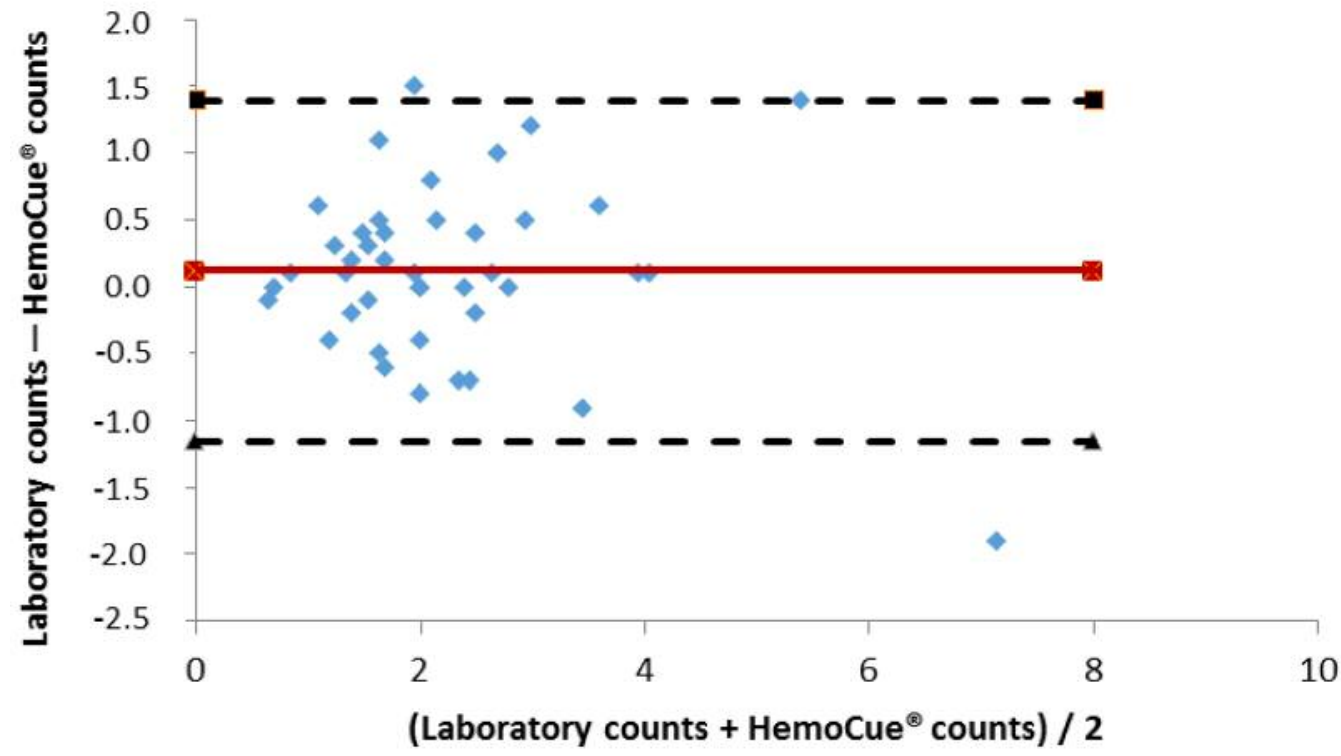

Figure 2. Bland-Altman plot of agreement of neutrophil counts between HemoCue WBC DIFF ${ }^{\circledR}$ and the University Hospital laboratory. Dashed lines represent the limits of agreement (average difference \pm 1.96-times the standard deviation of the difference), the red line indicates the mean difference.

laboratory up to about $2.5 \mathrm{~h}$ later. For WBC counts, the limits of agreement of $-1.61 \times 10^{9} / 1$ and $+2.34 \times 10^{9} / 1$ were wider than the clinically acceptable values of $\pm 1.0 \times 10^{9} / 1(5$ 7). For the neutrophil counts, the limits of agreement were also wider than clinically acceptable, i.e. $-1.15 \times 10^{9} / 1$ and $+1.39 \times 10^{9} / 1$ instead of $\pm 0.5 \times 10^{9} / 1$. Thus, the criteria for acceptable comparability between the methods were not met for WBC and for neutrophil counts. A difference of $1.0 \times 10^{9} / 1$ 
for WBC counts and of $0.5 \times 10^{9} / 1$ for neutrophil counts representing the step between two grades of toxicity according to CTCAE or more cannot be considered acceptable.

For the analyzers used at the laboratory, the coefficient of variation is 0.06 for the repeated measurements of both WBC and neutrophil counts, which agrees with those coefficients reported in the literature $(14,15)$. Since the repeatability standard deviations of the measurements at the laboratory should be small (mean of $4.65 \times 10^{9} / 1$ $\times 0.06=0.28 \times 10^{9} / 1$ for $\mathrm{WBC}$, and mean of $2.32 \times 10^{9} / 1$ $\times 0.06=0.14 \times 10^{9} / 1$ for neutrophils), and the correlation was substantial in this study for both WBC and neutrophil counts, the repeatability standard deviations of the HemoCue ${ }^{\circledR}$ WBC DIFF appeared too large to allow its use by patients and relatives at home. The differences between the cell counts obtained from the HemoCue ${ }^{\circledR}$ WBC DIFF at the patients' homes and those obtained from the laboratory may have relevant conseque1283nces for the patients in two ways. On the one hand, if the cell counts measured with the HemoCue ${ }^{\circledR}$ WBC DIFF at home are lower than those obtained from the laboratory, patients would not receive their chemotherapy although their WBC and neutrophil counts would allow them to be treated, which likely would have a negative impact on their prognoses. On the other hand, if the cell counts measured with the HemoCue ${ }^{\circledR}$ WBC DIFF at home were higher than those from the laboratory, the patients would not be able to receive chemotherapy and would have travelled to the hospital in vain. Thus, according to the preliminary results of this study, the use of the HemoCue ${ }^{\circledR}$ WBC DIFF at home appears not to be optimal and may not be recommended at this stage. However, the limitations of this pilot study have to be taken into account during the interpretation of its results, including the small number of patients and measurements, the fact that only one measurement was performed by the patients per session and the sometimes relatively long time interval between the measurement at the patient's home and the subsequent measurement at the laboratory [more than $120 \mathrm{~min}$ in $24 \%$ (10/42) of the measurements]. Moreover, almost all patients experienced problems with the lancet when taking blood samples during at least one of their measurements with the HemoCue ${ }^{\circledR}$ WBC DIFF. This may have contributed to the disagreement between methods due to the fact that the amount of blood was less than the required $10 \mu \mathrm{l}$ or partially clotted. In addition to the use of a different type of lancet, more intensive training, which was required by some patients, may also contribute to overcoming this problem. Therefore, the study will continue with a different type of lancet supplemented by intensive training of patients and relatives about its proper use.

Furthermore, it should be kept in mind that the HemoCue ${ }^{\circledR}$ WBC DIFF was developed to be used by healthcare professionals and not by patients and relatives. It may well be that the results would have been different, if, for example, nurses visiting the patients at their homes had performed the blood tests. However, this scenario appears only realistic if there are sufficient resources available to do so, including man-power. Otherwise, the patients would have to travel to hospital as before. It appears that the greatest benefit of the use of the HemoCue ${ }^{\circledR}$ WBC DIFF would be achieved if the device could be used by patients or relatives at home. This idea was also reflected by the results of the patient interviews. All 14 patients considered the use of the device at home a great advantage when compared to measurement at the laboratory, particularly because they would not have to travel to the laboratory and wait to receive their results.

In summary, given the limitations of this study, the criteria for acceptable comparability between the two methods were not met for WBC and neutrophil counts. Since the standard deviations of the HemoCue ${ }^{\circledR}$ WBC DIFF appeared too large, its use of the HemoCue ${ }^{\circledR}$ WBC DIFF at patients' homes may not be recommended at this stage. However, the preliminary findings of this study need to be verified in a larger cohort of patients using a different type of lancet supplemented by intensive training. Additional prospective studies are required to further evaluate the usability of the HemoCue ${ }^{\circledR}$ WBC DIFF by patients at home that should include a greater number of measurements by the patients on the same day and subgroup analyses regarding the time interval between the measurements at home and at the laboratory. Additional studies are desirable, particularly because all patients considered the use of the HemoCue ${ }^{\circledR}$ WBC DIFF at their homes a great advantage compared to measurement at a laboratory.

\section{Funding}

The study is part of the project InnoCan, which is funded by Interreg Deutschland-Danmark with funds from the European Regional Development Fund.

\section{Conflicts of Interest}

On behalf of all Authors, the corresponding Author states that there is no conflict of interest related to this study.

\section{References}

1 Stewart BW and Wild CW (eds.): World Cancer Report 2014. World Health Organization, International Agency for Research on Cancer, IARC Publications, 2014.

2 Osei-Bimpong A, Jury C, McLean R and Lewis SM: Point-ofcare method for total white cell count: an evaluation of the HemoCue WBC device. Int J Lab Hematol 31: 657-664, 2009.

3 Spaeth BA, Shephard MD, McCormack B and Sinclair G: Evaluation of HemoCue white blood cell differential counter at a remote health centre in Australia's Northern Territory. Pathology 47: 91-95, 2015. 
4 Karawajczyk M, Haile S, Grabski M and Larsson A: The HemoCue WBC DIFF system could be used for leucocyte and neutrophil counts but not for full differential counts. Acta Paediatr 106: 974-978, 2017.

5 Bui HN, Bogers JP, Cohen D, Nio T and Herruer MH: Evaluation of the performance of a point-of-care method for total and differential white blood cell count in clozapine users. Int J Lab Hematol 38: 703-709, 2016.

6 Bland JM and Altman DG: Statistical methods for assessing agreement between two methods of clinical measurement. Lancet 327: 307-310, 1986.

7 Bland JM and Altman DG: Measuring agreement in method comparison studies. Stat Meth Med Res 8: 135-160, 1999.

8 Giavarina D: Understanding Bland Altman analysis. Biochem Medica 25: 141-151, 2015.

9 National Institutes of Health/National Cancer Institute: Common Terminology Criteria for Adverse Events (CTCAE) version 5.0. U.S. Department of Health and Human Services, National Institutes of Health, National Cancer Institute, 2017. Available at https://ctep.cancer.gov/protocoldevelopment/electronic applications/docs/CTCAE_v5_Quick_Reference_8.5x11.pdf

10 Inoue Y, Yamashita N, Tokunaga E, Tanaka K, Ueo H, Saeki H, Oki E, Yamamoto H and Maehara Y: A locally advanced breast cancer that achieved pCR with pertuzumab, trastuzumab and docetaxel: Case report. Anticancer Res 37: 1917-1921, 2017.

11 Tanaka S, Iwamoto M, Kimura K, Takahashi Y, Fujioka H, Sato N, Terasawa R, Kawaguchi K, Ikari A, Tominaga T, Maezawa S, Umezaki N, Matsuda J and Uchiyama K: A phase II study of adjuvant chemotherapy of tegafur-uracil for patients with breast cancer with HER2-negative pathologic residual invasive disease after neoadjuvant chemotherapy. Anticancer Res 36: 6505-6509, 2016.
12 Waldrep AR, Avery EJ, Rose FF Jr., Midathada MV, Tilford JA, Kolberg HC and Hutchins MR: Breast cancer subtype influences the accuracy of predicting pathologic response by imaging and clinical breast exam after neoadjuvant chemotherapy. Anticancer Res 36: 5389-5395, 2016.

13 Jueckstock J, Burkhardt N, Kuhn C, Blankenstein T, Mahner S, Schindlbeck C, Janni W, Rack B and Mylonas I: Expression of activin during and after chemotherapy in peripheral blood of patients with primary breast cancer. Anticancer Res 36: 21532159, 2016.

14 Juul S, Pliskin JS and Fineberg HV: Variation and information in white blood cell differential counts. Med Decis Making 4: 6980,1984

15 Bundesärztekammer: Richtlinie der Bundesärztekammer zur Qualitätssicherung laboratoriumsmedizinischer Untersuchungen - Rili-BÄK. Dtsch Arztebl 111: A1583-A1618, 2014.
Received July 6, 2018

Revised July 16, 2018

Accepted July 19, 2018 